\title{
K.(U.)K. MILITÄRBIBLIOTHEKEN
}

\section{von Erik Gornik}

Zusammenfassung: Dieser Beitrag befasst sich aus einer quantitativen Perspektive mit den Militärbibliotheken der ehemaligen k.(u.)k. Armee, Der bibliothekshistorische Blick wird dabei basierend auf einer Bibliotheksstatistik aus dem Jahre 1870, in Form einer Momentaufnahme zurück getan. Nach einer Erläuterung zu dieser Statistik werden die darin erfassten Militärbibliotheken zusammenfassend vorgestellt. Zwei Übersichtsgrafiken sollen das Bild sowohl vom Umfang des Bibliotheksnetzes, als auch der Bibliotheksbestände abschließen.

Schlüsselwörter: Österreich-Ungarn; k.u.k. Armee; Bibliothek; Militärbibliothek; Bibliotheksbestand; Bibliotheksstatistik

\section{AUSTRIAN-HUNGARIAN MILITARY LIBRARIES}

Abstract: The article deals with the military libraries, housed by departments of the former Austrian-Hungarian Army. It both shows from a quantitative perspective, based on a library statistic of the year 1870, this statistic, as well as the number of military libraries and the scope of their library collection. This view is done in form of a snapshot of the military libraries which had been registered by the statisticians in 1870. At last two charts are shown, which illustrate the quantity of the existed libraries and their holdings.

Keywords: Austria-Hungary; Austro-Hungarian army; library; military library; library collection; library statistics

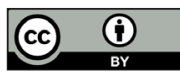

Dieses Werk ist lizenziert unter einer

Creative-Commons-Lizenz Namensnennung 4.0 International 


\section{Inhalt}

Einleitung

1. Die Bibliotheksstatistik der k. k. statistischen Zentralkommission

2. Die k.(u.)k. Militärbibliotheken

2.1. Amtsbibliotheken

2.2. Bibliotheken der militärischen Bildungsanstalten

2.3. Bibliotheken militärischer Versorgungs-, Heil- und Badeanstalten

2.4. Bibliotheken der Truppenkörper

2.5. Militärvereinsbibliotheken

3. Zusammenfassung

\section{Einleitung}

Das militärische Bibliothekswesen Österreich-Ungarns ist ein in großen Teilen unerforschtes Gebiet. Eigenständige Publikationen dazu konnten im Zuge der Recherchen für diesen Artikel nicht nachgewiesen werden. Die selbstständig erschienene Literatur zur österreichischen Bibliotheksgeschichte beantwortet speziell zu diesen Bibliotheken höchstens oberflächlich Fragen, und häufig werden lediglich in vereinzelten, unselbstständig erschienenen Werken, Militärbibliotheken zum Gegenstand der Betrachtung. ${ }^{1}$ Die vom Verfasser dieses Beitrags zusammenfassend so bezeichneten k.(u.)k. Militärbibliotheken bilden damit größtenteils ein bibliothekshistorisches Forschungsdesiderat. Zuerst ist hier natürlich die Frage von Interesse, was überhaupt existiert hat? Wie stellte sich das Bibliotheksnetz dar bzw. welche Dienststellen oder dem Militär nahe stehende Vereinigungen besaßen überhaupt Bibliotheken?

Der vorliegende Artikel versucht einen ersten bibliothekshistorischen Überblick über die Bibliotheken im österreichisch-ungarischen Militär zu geben. Wichtige Grundlage für diese Arbeit soll eine Quelle aus dem Jahre 1870 sein, genauer eine Bibliotheksstatistik der Statistikbehörde Österreichs, der sogenannten k.k. Statistischen Zentralkommission. ${ }^{2}$ Diese Statistik dürfte überhaupt die erste Bibliotheksstatistik Österreichs gewesen sein, die in einem derartigen Umfang durchgeführt wurde. Aufihrer Basis soll ein Überblick sowohl vom Umfang der Anzahl an Bibliotheken der k.(u.)k. Armee als auch deren Bestände zum Zeitpunkt der Datenerhebung skizziert werden. Das geschieht in Form einer Momentaufnahme mit dem Fokus auf das Jahr 1870 und beschränkt sich auf eine quantitative Darstellung der Bibliotheken und ihrer Bestände. 
Im begrenzten Rahmen dieses Artikels können nur beispielhaft ausgewählte Bibliotheken bzw. deren Dienststellen kurz besprochen werden. Einer organisatorisch-hierarchischen Vorgehensweise folgend, sind das jene die in der Statistik der Gruppe der Amtsbibliotheken zugeordnet worden sind. Alle anderen in der Statistik erfassten Militärbibliotheken finden in weiterer Folge eine zusammenfassende Bearbeitung gemäß ihrer Zuordnung zu einer der jeweiligen Bibliotheksgruppen, die von den Statistikern erstellt worden sind.

\section{Die Bibliotheksstatistik der k. k. statistischen Zentralkommission}

Im Jahr 1873 und 1874 erschien in zwei Heften eine Bibliotheksstatistik der k.k. Statistischen Zentralkommission in der (vermutlich) zum ersten Mal in größerem Umfang der Stand der Bibliotheken in Österreich erhoben wurde. Initiativen zur Durchführung einer derartigen Erhebung begannen zu Beginn des Jahres 1866, noch vor der politischen Zweiteilung der Habsburgermonarchie. Als diese aber mit dem österreichisch-ungarischen Ausgleich von 1867 in eine österreichische und eine ungarische Reichshälfte geteilt wurde, verzögerten sich vorerst weitere Arbeiten, die nach einiger Zeit ab 1870 nun wieder fortgesetzt werden konnten. Allerdings war die k.k. Statistische Zentralkommission nun eine rein cisleithanische Behörde geworden, konnte also nur die Bibliotheksdaten für die österreichische Reichshälfte erheben. ${ }^{3}$

Als zeitliche Grenze der Erhebung legte man den Stand der Bibliotheken zum 31.12.1870 fest. Die Ermittlung der Daten erfolgte mittels Formularen, die man an die diversen Bibliotheken und Behörden (Träger der Bibliotheken) versandte. Die Fragestellungen zur Datenerhebung auf diesen Formularen lassen sich in fünf Themenblöcke zusammenfassen: ${ }^{4}$

\section{Allgemeine Angaben}

Hierunter fallen Fragen zur Trägerorganisation der Bibliothek, dem Gründungsdatum, Öffnungszeiten und Entlehnfälle pro Jahr (für das Jahr 1870).

2. Bibliotheksbestand

In tabellarischer Form wurde die Anzahl der Medieneinheiten nach Fachgebieten (Theologie, Medizin, Philosophie usw.) getrennt abgefragt Vier Spalten erhoben die Anzahl von: Werken, Bänden, einzelnen Heften und einzelnen Blättern.

3. Personal

Dieser Block diente der Abfrage personeller Daten. So wurde, hierar- 
chisch getrennt sowohl Anzahl, als auch Gehalt und sonstige Bezüge von Vorstand, anderen Beamten und Bibliotheksdienern abgefragt. Darüber hinaus auch, wer die Ausgaben der Bibliothek bestritt bzw. von wem diese finanziert wurde.

4. Ausgaben

Für das Jahr 1870 sollten die Ausgaben der Bibliothek für Gehälter, Bücherankauf und Buchbinderkosten, sowie sonstige Aufwendungen abgefragt werden.

5. Sonstige Angaben

Das waren weitere Angaben die durch die Bibliothek bekannt zu geben waren. Beispielsweise „Angaben über Bücher [...] welche durch Seltenheit oder Schönheit [...] besonders bemerkenswerth sind. “5

Im Verlauf der Jahre 1871 und 1872 langten dann mehr als 600 der versendeten Erhebungsformulare von den diversen Bibliotheken wieder bei der k.k. Statistischen Zentralkommission ein. Die Daten wurden gesichtet, geprüft und von allen eingetroffenen Formularen blieben 526 Eingaben übrig, die man als tauglich für die Bibliotheksstatistik befand und die Datengrundlage für diese darstellten. Auf Basis dieser 526 Eingaben konnte man einen Bibliotheksbestand von mehr als 4,7 Millionen Medieneinheiten nachweisen. ${ }^{6}$ Die Bibliotheken wurden für die Statistik in sechs Gruppen eingeteilt:

1. Öffentliche Studien-, höhere Lehranstalts-, Instituts- und Mittelschulbibliotheken

2. Bibliotheken der geistlichen Kooperationen

3. Hof-, Staats-, Landes- und Gemeindebibliotheken

4. Privatbibliotheken

5. Militärbibliotheken

6. Vereinsbibliotheken

Für die weiteren Betrachtungen dieses Artikels ist die fünfte Gruppe, Militärbibliotheken, die maßgebliche. Bei dieser Gruppe ist zu beachten, dass die erwähnte Beschränkung des Tätigkeitsfeldes der k.k. Statistischen Zentralkommission auf die österreichische Reichshälfte nach dem Österreichisch-ungarischen Ausgleich nicht zum Tragen kam. Die Armee war im Sinne der Realunion eine gemeinsame Institution beider Staaten, womit auch Militärbibliotheken erfasst werden konnten, die in der ungarischen Reichshälfte lagen. 


\section{Die k.(u.)k. Militärbibliotheken}

Bibliotheken militärischer Dienststellen in der österreichischen bzw. habsburgischen kaiserlichen Armee lassen sich zeitlich schon recht früh nachweisen. Mit einem Gründungsdatum von $1752^{7}$ ist beispielsweise die Bibliothek der Theresianischen Militärakademie in Wiener Neustadt die älteste Militärbibliothek Österreichs. Von einem größeren oder gar weitverzweigten Netz an Bibliotheken bis in die Zeit nach 1815 kann aber nicht ausgegangen werden. Die meisten Militärbibliotheken dürften in der ersten Hälfte des 19. Jahrhunderts gegründet worden sein. ${ }^{8}$ In der Phase zwischen den französischen bzw. napoleonischen Kriegen und dem Österreichisch-ungarischen Ausgleich stieg die Zahl der Bibliotheksgründungen kontinuierlich an. Zum Zeitpunkt der Arbeiten und Erhebungen für die Bibliotheksstatistik erfasste man mit Stand zum 31.12.1870 hundert Militärbibliotheken, von denen 95 den Bestand von 287.533 Medien bekannt gegeben hatten (fünf der Bibliotheken konnten keine Angaben über den Umfang ihres Bibliotheksbestandes machen). ${ }^{9}$ Die Mehrzahl der erfassten Bibliotheken war in den 1850 er und 1860 er Jahren gegründet worden. ${ }^{10} \mathrm{Zu}$ beachten ist hier aber, dass es weit mehr als hundert militärische Dienststellen gab, von denen vermutlich auch einige ebenfalls Bibliotheken betrieben, diese Dienststellen jedoch „von der Zusendung der gewünschten Auskünfte über den Stand ihrer Büchersammlungen Umgang genommen haben."11 Festzustellen bleibt daher, dass in der Statistik sicherlich nicht alle Militärbibliotheken angeführt sind, die Österreich-Ungarns Armee betrieben hat.

Ebenso wie man für die Gesamtstatistik sechs Gruppen von Bibliotheken gebildet hat, wurde die Gruppe der Militärbibliotheken wiederrum unterteilt und zwar in fünf Gruppen, denen die hundert erfassten Bibliotheken zugeordnet wurden:

1. Amtsbibliotheken

2. Bibliotheken militärischer Versorgungs-, Heil- und Badeanstalten

3. Bibliotheken der Truppenkörper

4. Militärvereinsbibliotheken

5. Militärlehranstalten und Schulen

\subsection{Amtsbibliotheken}

Von den insgesamt hundert erhobenen Militärbibliotheken wurden sieben in die Gruppe der Amtsbibliotheken eingeteilt. ${ }^{12}$ Man kann wohl davon ausgehen, dass die Statistiker als Amtsbibliothek solche Bibliotheken ka- 
tegorisierten, die bei obersten militärischen Behörden (Reichskriegsministerium, Generalaten, höhere Kommanden u.dgl.) der k.(u.)k. Armee eingerichtet bzw. organisatorisch in deren Rahmen eingebunden waren. Sie dürften meist zur Benutzung durch die dortigen Bediensteten vorgesehen gewesen sein. Diese sieben Bibliotheken waren bei folgenden Dienststellen angesiedelt:

- Kriegsbibliothek

- Technisches und administratives Militärkomitees

- Marinesektion des Reichskriegsministeriums

- Marinebibliothek

- Garnisonsbibliothek in Graz

- Garnisonsbibliothek in Krakau

- Generalstabsabteilung des Generalkommandos Lemberg

\subsubsection{Die Kriegsbibliothek}

Die Kriegsbibliothek und spätere Bibliothek des Wiener Kriegsarchivs geht zurück auf das Jahr 1776, wo sie als Bibliothek des Hofkriegsrätlichen Archivs gegründet wurde. ${ }^{13}$ Sie zählt damit zu den ältesten Militärbibliotheken Österreichs. Eine recht günstige Entwicklung verdankte sie der Einflussnahme des Feldmarschalls Radetzky, der eine Bibliothek schaffen wollte, die dem Offizierskorps der Armee offenstehen und ein breites Spektrum an Wissenschaftsgebieten abdecken sollte. Ab 1808 als selbstständige Abteilung im Kriegsarchiv geführt, erfolgte im Jahr 1811 die Umbenennung auf den Namen Kriegsbibliothek. ${ }^{14}$ In den 1820er Jahren wurde bereits ein Bestandskatalog, ${ }^{15}$ aber 1853 dann der erste alphabetisch angelegte Katalog publiziert. In diesem war bereits ein Bestand von 20.000 Medien nachgewiesen. Organisatorisch gehörte die Bibliothek zum Kriegsarchiv das bis 1918 als Hilfsorgan dem k.u.k. Reichskriegsministerium unterstellt war. Zum Zeitpunkt der Statistikerhebung hatte die Kriegsbibliothek 35.057 Medien in ihrem Bestand und war damit die an Beständen größte Bibliothek innerhalb der k.(u.)k. Armee. Eine führende Rolle, die sie (nach bisherigem Recherchestand) bis zur Auflösung der Monarchie im Jahre 1918 behalten sollte. ${ }^{16}$

\subsubsection{Die Bibliothek des Technischen und administrativen Militärkomitees}

Im Jahr 1869 schuf man das sogenannte k.u.k. Technische und administrative Militär-Comité. Hauptaufgaben dieses Komitees waren neben dem Verfolgen des allgemeinen wissenschaftlichen und technischen Fortschritts (mit 
Fokus auf die Kriegsverwendbarkeit), die Durchführung von Versuchen und Studien bzw. die Erstellung wissenschaftlicher Beiträge, Dienstbücher und Vorschriften für die k.u.k. Armee. Darüber hinaus hatte es auch die Zuständigkeit, Begutachtungen der Befestigungsbauten Österreich-Ungarns durchzuführen, Empfehlungen dazu abzugeben und die höhere Fortbildung für Offiziere technischer Waffengattungen in Form von Lehrgängen zu organisieren. ${ }^{17}$ Das Komitee bestand aus dem Präsidium und vier Sektionen und war dem Reichskriegsministerium unterstellt. Die Bibliothek war beim Präsidium angesiedelt und wies Ende 1870 einen Bestand von 16.616 Medien auf.

\subsubsection{Die Marinebibliothek(en)}

Die Marinebibliothek und die Bibliothek der Marinesektion des k.u.k. Reichskriegsministeriums sind gemeinsam zu betrachten, da sie in fachlicher Hinsicht eng kooperierten. Durch ihre Dislozierung in Pola ${ }^{18}$ zwar räumlich von Wien (und damit der Zentralstelle) weit entfernt, entwickelte sie sich dennoch ab Mitte der 1870er Jahre zu einer Art Zentralbibliothek für das Marinewesen innerhalb der k.u.k. Armee.

Die Marinesektion war nach dem österreichisch-ungarischen Ausgleich 1867 als Teil des k.u.k. Reichskriegsministeriums eingerichtet worden, die Marinebibliothek geht in ihren Ursprüngen bis auf das Jahr 1802 zurück. In der Bibliotheksstatistik wies man für die Marinesektion 1.189 und für die Marinebibliothek 4.750 Medien nach.

Was in der Bibliotheksstatistik 1870 aber noch getrennt angeführt und wohl auch so eingemeldet worden ist, vereinigte man gegen Ende des 19. Jahrhunderts, als die Bestände der Marinesektion und jene der Marinebibliothek administrativ in Pola zusammengeführt und zentral von der Marinebibliothek verwaltet wurden. ${ }^{19}$

\subsubsection{Die Garnisonsbibliotheken Graz und Krakau}

Graz und Krakau waren jeweils Standorte größerer Militärterritorialbehörden und anderer militärischer Dienststellen. Graz war unter anderem Sitz des sogenannten Generalates für Steiermark, Kärnten, Krain, Istrien, Görz, Gradisca und die Stadt Triest und in Krakau war neben anderen militärischen Einheiten, das XII. Truppendivision- und Militärkommando zu Krakau disloziert, dass zum Generalat für Galizien und die Bukowina gehörte. ${ }^{20}$

Für die Garnisonsbibliothek Graz ließen sich bislang keine Nachweise selbstständiger Publikationen (Kataloge u.dgl.) finden. Anzunehmen ist, 
dass sie im Jahr 1867 errichtet worden war. ${ }^{21}$ In der Bibliotheksstatistik wurde für sie ein Bestand von 1.494 Medien erhoben, womit sie ähnlich umfangreich wie die Garnisonsbibliothek Krakau gewesen ist. Für letztgenannte haben sich Statuten erhalten. Auch wenn diese aus dem Jahr 1895 stammen, kann man davon ausgehen, dass der darin festgelegte Zweck der Bibliothek auch schon in ähnlicher Form im Jahre 1870 bestand. Sie diente demnach dazu „, die Erweiterung der militärische Kenntnisse und der allgemeinen Bildung zu ermöglichen [und] auch in Büchern belletristische Inhaltes eine Unterhaltungs-Lektüre zu bieten."22 Die Bibliothekstatistik wies für die Krakauer Garnisonsbibliothek einen Bestand von 1.174 Medien nach.

\subsubsection{Die Bibliothek der Generalstabsabteilung des Generalkommandos Lemberg}

Die k.u.k. Armee verfügte über mehrere Militärterritorialbehörden, deren Hauptsitz meist in einem der größeren Orte der Monarchie lag, wozu auch Lemberg zählte. In der Betrachtung ist die Tatsache interessant, dass die Generalstabsabteilung des Generalkommandos zu Lemberg ebenfalls ihre Bestände der k.k. Statistischen Zentralkommission meldete. Das Generalkommando Lemberg gehörte nämlich, wie jenes vorhin besprochene aus Krakau, ebenfalls zum Generalat für Galizien und die Bukowina. Obwohl man demnach im vorgesetzten Kommando in Lemberg scheinbar sehr diensteifrig an der Bibliotheksstatistik mitgewirkt hat, konnte für Lemberg bislang keine eigene Garnisonsbibliothek nachgewiesen werden. Den Bestand der Bibliothek der Generalstabsabteilung hat man mit 294 Medien angegeben. Es handelte sich demnach vermutlich um eine reine Dienststellen- bzw. kleine Amtsbibliothek, die für den dortigen Dienstgebrauch bestimmt war.

\subsection{Bibliotheken der militärischen Bildungsanstalten}

Für insgesamt dreizehn Schulen und Akademien der k.(u.)k. Armee wurden in der Statistik Bibliotheken nachgewiesen. Es dürften aber bei weitem nicht alle militärischen Ausbildungsanstalten die Bibliotheken unterhielten damit erfasst sein. Das Militär betrieb von der Olmützer Kadettenkompanie, über die Genieakademie Znaim bis hin zu den Soldatenknaben-Erziehungshäusern zahlreiche Bildungsanstalten, von denen einige potentielle Träger von Bibliotheken gewesen sein könnten. ${ }^{23}$ Andere Einrichtungen dürften wiederrum keine eigenen Bibliotheken besessen, sondern bestehende Bibliotheken an ihrem jeweiligen Standort genutzt haben, wie beispielsweise das Equitationsinstitut in Wiener Neustadt vermutlich die Bibliothek der (Theresianischen) Militärakademie nutzte. 
Die von den Statistikern erhobenen Bibliotheken waren bei folgenden Bildungseinrichtungen: ${ }^{24}$

- Technische Militärschule (Mährisch Weisskirchen) - zwei Bibliotheken

- Technischen Militärakademie (Wien)

- Militärakademie Wiener Neustadt (Wiener Neustadt)

- Medizinisch-chirurgische Josephs-Akademie (Wien)

- Militärtierarznei Institut (Wien)

- Militärgeographisches Institut (Wien)

- Militärkollegium (St. Pölten)

- Kriegsschule (Wien)

- Kadettenschule (Lemberg)

- Nautische Schule (Lussin piccolo ${ }^{25}$ )

- Nautische Schule (Cattaro ${ }^{26}$ )

- Marineunterrealschule (Pola)

Unter allen fünf Gruppen, in die man die Militärbibliotheken einteilte, hatten die Bibliotheken der militärischen Bildungsanstalten zusammen 117.475 Medien in ihrem Bestand. Damit bildeten sie insgesamt die an Beständen größte Gruppe. ${ }^{27}$

\subsection{Bibliotheken militärischer Versorgungs-, Heil- und Badeanstalten}

Das militärische Sanitätswesen der k.(u.)k. Armee war breit gestreut und ab dem Jahr 1870 wurde sogar ein eigenes militärstatistisches Jahrbuch publiziert, das mit Schwergewicht Daten zum Militärsanitätswesen erhob. ${ }^{28}$ Eine Vielzahl an derartigen Einrichtungen stand für die Patienten zur Verfügung. Alleine die Zahl an Kuranstalten dürfte wohl mehr als 50 Institutionen betragen haben, die von Sutinsko bis Kaltenleutgeben verschiedene Formen der Heilbehandlung anboten. ${ }^{29}$ In diesen Anstalten standen im Jahr 1870 mehr als 158.000 Armeeangehörige in Behandlung. ${ }^{30}$ Es kann wohl davon ausgegangen werden, dass annähernd alle größeren Heil- und Sanitätsanstalten bzw. Militärspitäler auch Bibliotheken betrieben haben, um ihre Patienten mit Lektüre zu versorgen. In administrativen Vorschriften der 1880 er Jahre sind auch vereinzelt Bestimmungen für Spitalsbibliotheken nachzuweisen. ${ }^{31}$ Umso erstaunlicher ist es, dass von der Vielzahl an potentiellen Bibliotheksträgern lediglich sechs Daten an die k.k. Statistische Zentralkommission gemeldet haben und zwar:

- Militärbadeheilanstalt (Baden)

- Wiener k. k. Invalidenhaus

- Garnisonsspital (Graz) 
- Garnisonsspital (Prag)

- Garnisonsspital (Theresienstadt)

- Garnisonsspital (Triest)

Für diese Bibliotheken wurde ein Bestand von 8.516 Medien angegeben. In dieser Angabe enthalten ist aber der Bestand von 763 „Werken“ enthalten, die vom Garnisonsspital Prag gemeldet wurden. ${ }^{32}$ Hier finden sich keine Angaben zur Anzahl der Medien. Die Zahl von 763 ist also nicht der Anzahl der Medien entsprechend, die vermutlich höher ausfallen dürfte.

\subsection{Bibliotheken der Truppenkörper}

Die Bibliotheken der Truppenkörper (also v.a. Infanterie-, Kavallerie-, Artillerieregimenter) waren jene, die in Summe die zahlreichsten waren. Von allen in der Statistik angeführten hundert Militärbibliotheken sind fast dreiviertel sogenannte Regimentsbibliotheken. Die meisten Regimentsbibliotheken entstanden in der ersten Hälfte des 19. Jahrhunderts. Eines der frühesten Beispiele wäre die Bibliothek des Infanterieregiments Graf von Lacy Nr. 22, die rund um das Jahr 1803 entstanden sein dürfte. Der Oberst dieses Regiments „lud die Officiere zur Stiftung einer RegimentsBibliothek mit dem Vorschlage ein, daß jeder etwas von seinem Büchervorrathe, und dann zu Unterhaltung und Vermehrung derselben auch etwas im Gelde beytragen möchte. " ${ }^{33}$ Im Laufe der Zeit wurden dann zahlreiche Regimentsbibliotheken eingerichtet und gegen Ende des 19. Jahrhunderts konnte man schließlich konstatieren, dass beinahe jedes „Regiment, sogar jedes selbstständige Bataillon [...] seine eigene Truppen-Bibliothek [besitzt]. “34 Die Regimentsbibliotheken wurden von den Gagen der Offiziere unterhalten, standen damit also auch in deren Eigentum.

Für diese Bibliotheksgruppe wurde zusammenfassend ein Bestand von 94.207 Medien nachgewiesen, was sie in quantitativer Hinsicht zur zweitgrößten Gruppe machte. ${ }^{35}$

\subsection{Militärvereinsbibliotheken}

Nach dem Vorbild ziviler wissenschaftlicher Gesellschaften wurde Mitte 1867 in Wien ein militärwissenschaftlicher Verein gegründet. Dieser Militär-wissenschaftlicher Verein Wien, auch Militär-Casino Wien genannt, war die erste Vereinigung in der Armee ihrer Art, in der das Freizeitangebot für Offiziere (Kasino) mit einem (militär-)wissenschaftlichen Zweck verbunden wurde. ${ }^{36}$ Dem Wiener Beispiel sollten bis zum Ende der k.u.k. Monarchie 
noch zahlreiche weitere Vereinsgründungen folgen. Bei all diesen Vereinen richtete man auch Bibliotheken ein und so konnte in den späten 1880er Jahren festgestellt werden, dass die „militär-wissenschaftlichen Vereine über mitunter recht ansehnliche Büchersammlungen “37 verfügten. In der Bibliotheksstatistik von 1870 scheinen aber erst lediglich zwei Vereinsbibliotheken auf. Das ist die des Wiener Militärkasinos mit einem doch beachtlichen Bestand von 4.089 Medien, den man in nur drei Jahren nach der Gründung zusammengetragen hatte. Überdies wurde die Bibliothek des ehemals bestandenen Militärkasinos in Verona (Venetien musste nach dem verlorenen Krieg von 1866 an Frankreich abgetreten werden) in der Bibliothek des Wiener Militärkasinos aufgestellt. In der Statistik ist diese Bibliothek aber noch extra, mit einem Bestand von 3.435 Medien, angeführt. ${ }^{38}$ Offenbar wurde sie durch das Wiener Militärkasino getrennt administriert (jedenfalls noch 1870) und den Statistikern auch so gemeldet.

Zusammen wies diese vom Umfang her kleinste Gruppe der Militärbibliotheken einen Bestand von 7.524 Medieneinheiten auf.

\section{Zusammenfassung}

Hundert k.(u.)k. Militärbibliotheken erfasste die k.k. Statistische Zentralkommission in dieser ersten umfangreichen österreichischen Bibliotheksstatistik. Diese Militärbibliotheken wiesen zum 31. Dezember 1870 einen Bestand von annähernd 290.000 Medien auf. Die Statistiker hatten die Bibliotheken in fünf Hauptgruppen eingeteilt. Der Umfang der Bibliotheksbestände $^{39}$ den 95 von den hundert Bibliotheken ${ }^{40}$ getrennt nach den Hauptgruppen aufwiesen, stellt sich (bei insgesamt 287.533 Medien) so dar:

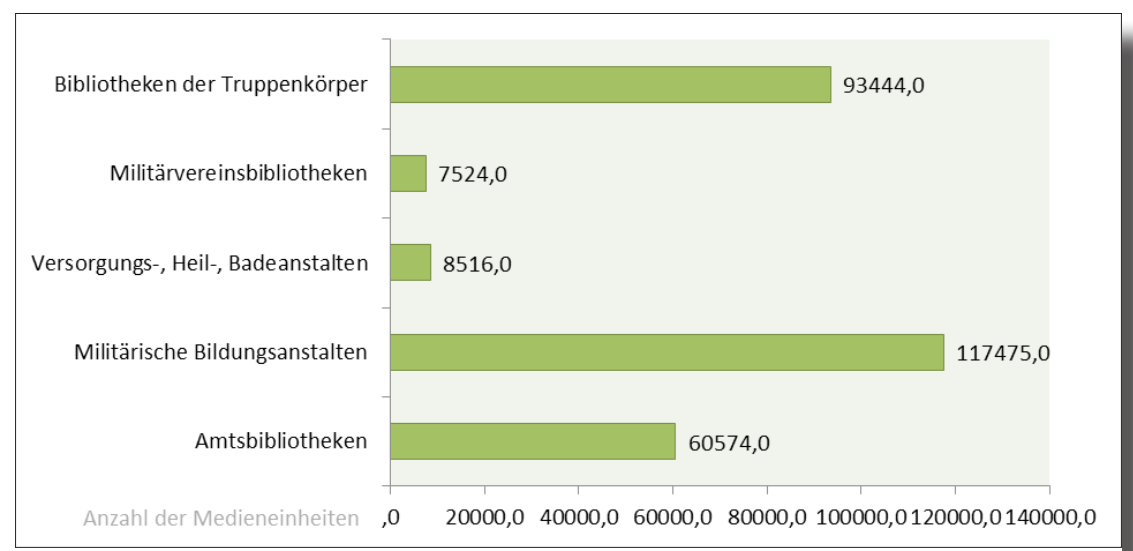


Damit wird nochmal deutlich, dass die militärischen Bildungsanstalten zusammen den größten Bestand aufwiesen. Demgegenüber rangiert als kleinste Gruppe die der Militärvereinsbibliotheken, wovon im Jahr 1870 zwei erfasst wurden. Die k.(u.)k. Militärbibliotheken waren mit Stichtag zum 31.12.1870, grafisch dargestellt, so verteilt:

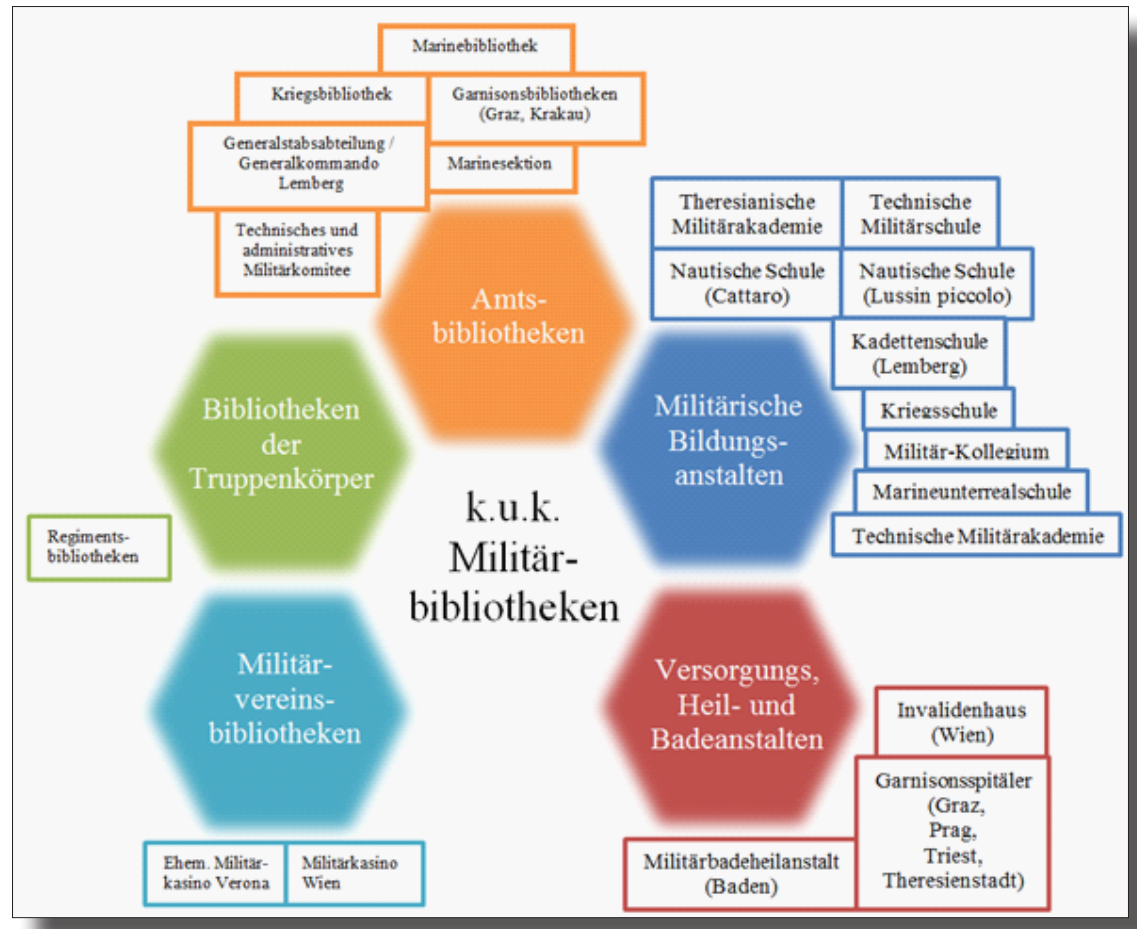

Dieser Artikel sollte einen Überblick zu der bisher recht unerforschten Thematik der Militärbibliotheken in der k.(u.)k. Armee geben. Nachdem nun ein erstes (nicht erschöpfendes) Was skizziert wurde, wäre natürlich auch das Wie von Interesse, also Fragestellungen aus qualitativer Perspektive wie Bestandsanalysen, Benutzergruppen u. dgl. Dies gilt natürlich nicht nur für die militärischen, sondern auch für die zivilen Bibliotheken die, in der als Quelle so interessanten, Bibliotheksstatistik von 1870 erfasst wurden und von denen einige heute nicht mehr existieren. Konnte dieser Artikel über seinen Informationsgehalt hinaus Kolleginnen und Kollegen auch zu bibliothekshistorischen Forschungen dazu motivieren, hat er seinem Zweck mehr als entsprochen. 
Erik Gornik, MA Bibliothek des Heeresgeschichtlichen Museums E-Mail: e.gornik@hgm.at

\section{Literatur}

Apfelbauer, Richard W.: Die Bibliothek des Wiener Kriegsarchivs. Ihre historische wie gegenwärtige Bedeutung, Wien 1981.

F.J.B.: Die Sanitäts-Verhältnisse der k. k. Armee im Jahre 1870. In: Österreichische Militärische Zeitung, 1874 / Heft 3, S. 181-195.

Gaisbauer, Adolph: Die Bibliothek des Kriegsarchivs. Eine Momentaufnahme mit Schlussfolgerungen. In: Mitteilungen des Österreichischen Staatsarchivs; Bd. 49, Wien 2001, S. 213-219.

[Garnisonsbibliothek Graz]. In: Fremdenblatt Nr. 1 / 21. Jg. vom 1. Jänner 1867, S. 3.

Inländische Begebenheiten. In: Wiener Zeitung vom 17.12.1803, S. 4758. Jobst, Johann (Hrsg.): Die Neustädter Burg und die K. u. K. Theresianische Militärakademie. Ein Führer in militärischer und kunstgeschichtlicher Beziehung. Wien; Leipzig 1908.

Kais. Königl. Militär-Schematismus für 1871, Wien 1871.

Militär-Casino Wien: Statuten für das Militär-Casino in Wien, Wien 1868.

Kais. Königl. Militär-Schematismus für 1871, Wien 1871.

Pizzala, Josef: Stand der Bibliotheken der im Reichsrathe vertretenen Königreiche und Länder zu Ende des Jahres 1870 / I. Theil, Wien 1873 (Mittheilungen aus dem Gebiete der Statstik; Band 20 / Heft 2).

Pizzala, Josef: Stand der Bibliotheken der im Reichsrathe vertretenen Königreiche und Länder zu Ende des Jahres 1870 / II. Theil, Wien 1874 (Mittheilungen aus dem Gebiete der Statstik; Band 20 / Heft 6).

Schmid, Ferdinand: Das Heeresrecht der Österreichisch-Ungarischen Monarchie, Wien; Leipzig 1903.

Statuten der Garnisons-Bibliothek zu Krakau, Krakau 1895.

Ueber Officiers-Bibliotheken. In: Militär-Zeitung Nr. 40 / 46. Jg. vom 6.7.1887, S. [319]-320.

Wagner, Walter: Zur Geschichte der k. und k. Marinebibliothek, In: Mitteilungen des Österreichischen Staatsarchivs; Bd. 15, Wien 1962, S. 336-389.

Wick, Ludwig: Die Militär-Bade- und Trinkkuranstalten. Anstalten des Ärars, des weißen Kreuzes und sonstige Widmungen, Wien 1903. 
1 Zur österr. Bibliotheksgeschichte u.a. Bick, Josef: Die staatlichen Bibliotheken Österreichs 1848-1948, Wien 1948; Pongratz, Walter: Abriß der Bibliotheksgeschichte. Mit besonderer Berücksichtigung Österreichs, Wien 1977 (Skripten für die österreichische Bibliothekarausbildung; 2); Stummvoll, Josef: Austrian libraries past and present, Chicago 1950; Unterkirchner, Franz: Die Bibliotheken Österreichs in Vergangenheit und Gegenwart, Wiesbaden 1980.

2 Pizzala / k.k. Statistische Central-Kommission (1873 u. 1874).

3 Vgl. Pizzala (1873), S. 2.

4 Vgl. ebda., S. 5f.

5 Ebda., S. 7.

6 Vgl. ebda., S. 3. Eine Gegenprüfung der erhobenen Zahlen durch weitere Recherchen konnte im Zuge dieses Artikels nicht durchgeführt werden. Kritisch ist aber unter anderem zu betrachten, dass vom bibliotheksinternen Personal selbst die erhobenen Zahlen eingemeldet wurden und nicht beispielsweise von externen Personen.

7 In der Bibliotheksstatistik wird 1752 als Gründungsjahr der Bibliothek angegeben. Andere Publikationen nennen das Jahr 1756. Vgl. Pizzala (1874), S. 38 und u.a. Jobst (1908), S. 278f. Die Gründung der Militärakademie erfolgte auf Befehl Maria Theresias am 14. Dezember 1751, vgl. u.a. Svoboda, Johann: Die Theresianische Militär-Akademie zu Wiener-Neustadt und ihre Zöglinge. Von der Gründung der Anstalt bis auf unsere Tage, Wien 1894 (3 Bände), S. XLIIf.

8 Vgl. Schmid (1903), S. 414.

9 Vgl. Pizzala (1874), S. 23 u. 43.

10 Vgl. ebda., S. 32.

11 Ebda., S. 23.

12 Vgl. Ebda., S. 38.

13 Vgl. Apfelbauer (1981), S. 10.

14 Vgl. Geisbauer (2001), S. 216.

15 Catalog über die in der k.k. Kriegs-Bibliothek befindlichen gedruckten Werke, Wien 1825.

16 Vgl. Pizzala (1874), S. 38.

17 Vgl. Normalverordnungsblatt für das k.k. (k.(u.)k.) Heer, 57. Stk. 1869, 63. Stk. 1873, 52. Stk. 1894.

18 Das heutige Pula in Kroatien.

$19 \mathrm{Vgl}$. Wagner (1962), S. 385.

20 Vgl. u.a. Militär-Schematismus (1871), S. 99 u. $106 \mathrm{ff}$.

$21 \mathrm{Vgl}$. Garnisonsbibliothek Graz (1867), S. 3.

22 Statuten Krakau (1895), §1. 
23 Vgl. dazu u.a. Rechberger von Rechkron, Josef: Das Bildungswesen im Österreichischen Heere vom dreissigjährigen Kriege bis zur Gegenwart, Wien 1878.

24 Vgl. Pizzala (1874), S. 42.

25 Das heutige Mali Lošinj in Kroatien.

26 Das heutige Kotor in Montenegro.

27 Vgl. Pizzala (1874), S. 31.

28 Vgl. Militär-statistisches Jahrbuch, Wien 1870-1904. 1907. 1911 (Ab dem Jg. 1895: Statistik der Sanitätsverhältnisse der Mannschaft des k.(u.)k. Heeres, Ab 1907: Sanitätsstatistischer Bericht d. k.(u.)k. Heeres).

29 Vgl. u.a. Wick (1903).

30 Vgl. F.J.B. (1870), S. 184.

$31 \mathrm{Vgl}$. u.a. Instruction für die Material-Verrechnung in den k. k. MarineSpitälern, Wien 1884, (§3: Führung des Bibliotheks-Kataloges und Verfassung des Bibliotheks-Veränderungs-Ausweises).

32 Vgl. Pizzala (1874), S. 38.

33 Inländische Begebenheiten (1803), S. 4758.

34 Ueber Officiers-Bibliotheken (1887), S. [319].

35 Vgl. Pizzala (1874), S. 29.

36 Vgl. Militär-Casino Wien (1868), §§ 1 und 2.

37 Ueber Officiers-Bibliotheken (1887), S. [319].

38 Vgl. Pizzala (1874), S. 44.

39 Zu beachten ist hier, dass bei zwei Bibliotheken nicht die Anzahl an Medien, sondern nur jene von Werken enthalten ist (Garnisonsspital Prag = 763, Infanterieregiment Nr. 5 = 236).

40 Fünf konnten wie in Kap. 2 erwähnt keine Angaben zu ihren Beständen machen (s. EN 10). 